\title{
The study of PSO-RBF neural network generalized predictive control strategy in unit plant
}

\author{
WANG HUI ${ }^{1, a}$, LING Hujun ${ }^{2, b}$, PAN LEI $^{3, c}$ \\ ${ }^{1}$ school of electric power, inner mongolia university of technology, huhhot 010080, China. \\ ${ }^{2}$ inner mongolia university of technology, huhhot 010080, China.

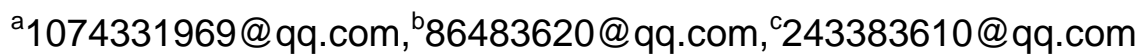

Keywords: particle swarm optimization algorithm RBF neural network generalized predictive control generating unit

\begin{abstract}
Unit coordinated control in thermal power plants is a system which is complex,nonlinear and is difficulty to establish accurate model, So it is hard to make system gain optimum running effect with conventional control strategy. PSO-RBF neural network is used to identify the mathematical model of coordinated control system and acts as a predictive model in generalized predictive control strategy, which is to achieves predictive control with online rolling optimization and real time feedback revision. Simulation results show that it has a strong robustness when the load condition changes,or big lag affects.
\end{abstract}

\section{Introduction}

Unit coordinated control in thermal power plants is a system which is strongly non-linear,strongly coupling and which has problems with large time delay,time-varying parameters,great inertia,big interference and so on,so it is difficult to build a accurate mathematical model. $^{[1]}$

Radial basis function neural network has a good capacity of local approximation and has some convergence performance.however,it has some defects like definiting hidden-layer center and width value.Particle swarm optimization algorithm has a good ability of global convergence, and the algorithm is easy to achieve.in this article,it makes PSO algorithm and RBF neural network combine to form a training algorithm which is called PSO-RBF to be the prediction model.

Generalized predictive control based on parametric model was proposed by Clarke in 1984. It is composed of model prediction,rolling optimization and feedback correction. It is based on generalized minimum variance control,and introduces the idea of multi-step prediction in optimization, the capability which includes anti-disturbance,random noise,delay time variation is markedly increased. ${ }^{[2]}$

This paper combines PSO-RBF neural network as the prediction model with GPC in the large-scale unit coordinated control system. in the end, the paper makes systemic simulation ,when the system varying condition,varying parameters and consisting big-lag,and simulation result indicates the algorithm has strong robustness.

\section{RBF neural network and PSO algorithm}

RBF neural network is a three-layer feed forward network with a single hidden layer as shown in fig.1. here $\left[x_{1}, x_{2}, \cdots, x_{n}\right]$ denotes the neural input values, $\left[c_{1}, c_{2}, \cdots, c_{h}\right]$ denotes center values of Gaussian Function. $\left[b_{1}, \cdots, b_{m}\right]$ denotes threshold values, $\left[y_{1}, y_{2}, \cdots, y_{m}\right]$ denotes output values.n,h,m respectively denotes numbers of input ,hidden ,output nodes.output expression are given:

$$
y_{i}=\sum_{j=1}^{h} \omega_{i j} \varphi\left(X, C_{i}\right)+b_{k}, i=1,2, \cdots, n, k=1,2, \cdots, m
$$

Where $\omega_{\mathrm{ij}}$ denotes the weights between the neuron and other neurons. 


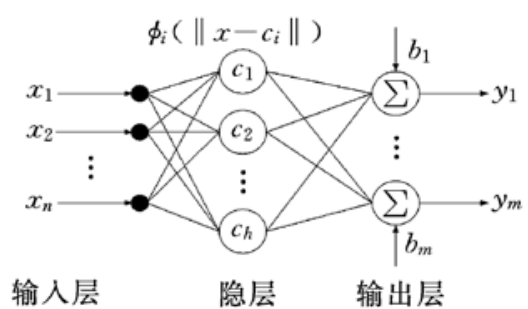

Fig.1 RBF neural network structure

Particle swarm optimization algorithm is a new global optimization algorithm based on swarm intelligence.its thoughts come from the reasearch on bird flock preying behavior.every particle represents potential solution of optimization problems.the degree or grade of excellence of solution was determined from fitness function.every particle update the velocities and the positions according to the following formula ${ }^{[3]}$.

$$
\begin{gathered}
v_{i d}^{t+1}=\omega v_{i d}^{t}+c_{1} r_{1}\left(p_{i d}-x_{i d}^{t}\right)+c_{2} r_{2}\left(p_{g d}-x_{i d}^{t}\right) \\
x_{i d}^{t+1}=x_{i d}^{t}+v_{i d}^{t+1}
\end{gathered}
$$

Where $t$ is iteration, $v t$ id is flight velocity which the th generation particle $\mathrm{i}$ was in the dth dimension space, $x \mathrm{t}$ id is position which the tth generation particle $\mathrm{i}$ was in the dth dimension space. $r_{1}, r_{2}$ are random numbers from 0 to $1 . \omega$ is inertia gene.

There are some problems in the following ways when PSO optimizing basic parameters of RBF neural network ${ }^{[4]}$.

(1) coding scheme of parameter.the dimension of particle is composed of RBF parameter to be optimized.particle dimension formula and encoding structure are given respectively as follows.

$$
\begin{aligned}
& D=n h+h+h m+m \\
& c_{11} c_{12} \cdots c_{1 n}, c_{21} c_{22} \cdots c_{2 n}, \cdots, c_{h 1} c_{h 2} \cdots c_{h n}, \sigma_{1} \sigma_{2} \cdots \sigma_{h}, \\
& \omega_{11} \omega_{12} \cdots \omega_{1 h}, \omega_{21} \omega_{22} \cdots \omega_{2 h}, \cdots, \omega_{m 1} \omega_{m 2} \cdots \omega_{m h}
\end{aligned}
$$

(2) selection of fitness function.define it as follows.

$$
\begin{gathered}
\boldsymbol{f}_{i}=\frac{\mathbf{1}}{\boldsymbol{R}_{\boldsymbol{i}}} \\
\boldsymbol{R}_{\mathrm{i}}=\frac{1}{N} \sum_{i=1}^{N}\left(\hat{y}_{i}-y_{i}\right)^{2}
\end{gathered}
$$

Where $\hat{y}_{i}$ is desired output values, $y_{i}$ is acual output values. $\mathrm{N}$ is the number of particle.

\section{PSO-RBF neural network generalized predictive control}

in the generalized predictive control,at time $\mathrm{t}=\mathrm{T}$, the optimization performance index is showed as equation $^{[5]}$ :

$$
J=\frac{1}{2} \sum_{i=d}^{N_{1}}\left[y_{r}(t+i)-y_{p}(t+i)\right]^{2}+\frac{1}{2} \sum_{j=1}^{N u} \lambda_{i}[\Delta u(t+j-1)]^{2}
$$

Where where $N_{1}$ is the maximum prediction time domain, $N_{\mathrm{u}}$ is the control time domain, $\lambda_{\mathrm{i}}$ is the weighting constants. $\Delta u$ is the control increment, $y_{r}(\mathrm{t})$ is the setting value. $y_{p}(\mathrm{t}+\mathrm{i})$ is the output of the fuzzy neural network model.

The performance index function is optimized at each sampling time using a gradient descent method, then control law $\Delta u$ can be drawn. Based on the rolling optimization of the generalized predictive control, the control variables can be selected by:

$$
u(t+1)=u(t)+[1,0, \cdots, 0](I+\mu \lambda)^{-1} \mu \delta y_{u} e
$$

where $\mu$ is the optimization step when calculating $\Delta u$ with the way of the gradient descent method. $\delta y_{u}$ is worked out by the trained neural network weights and the derivative of sigmoid function. $e$ is prediction output error.

Due to time-varying, nonlinear and various random interference inevitable, therefore the output of 
the prediction model can not be completely consistent with the actual object, thus it need to be corrected by the feedback prediction model. The error between the actual output and the model output is:

$$
e_{m}(t)=y(t)-y_{m}(t)
$$

the predicted output is:

$$
y_{p}(t+i)=y_{m}(t+i)+h e_{m}(t)
$$

where, $\mathrm{h}$ is the error correction coefficient.

The steps of algorithm of PSO-RBF neural network generalized predictive control online are summarized as follows:(1)initialize PSO-RBF neural network and the controller parameters; (2)sample input and output data of controlled object and correct weights of neural network model according to algorithm of PSO-RBF;(3)work out control rate according to equation; (4)return step (2).

\section{Simulation}

\section{PSO-RBF neural network error curve and generalization curve}

Coordinated control system is a dual-input,dual-output coupling complex control object.the two inputs of the object are:steam turbine opening $\mu_{\mathrm{T}}$ and boiler combustion rate $\mu_{\mathrm{B}}$, the two outputs are:power output $\mathrm{N}_{\mathrm{e}}$ and main steam pression $\mathrm{P}_{\mathrm{t}}$.the transfer functions of $300 \mathrm{MW}$ power unit at $70 \%, 100 \%$ are as follows:

$$
\begin{aligned}
& {\left[\begin{array}{l}
N_{E} \\
P_{T}
\end{array}\right]=\left[\begin{array}{cc}
\frac{4.665 s(99 s+1)}{\left(528 s^{2}+50 s+1\right)(4.1 s+1)} & \frac{2.069(311 s+1)}{(149 s+1)^{2}(22.4 s+1)} \\
-1.42\left(0.04+\frac{0.96}{70 s+1}\right) & \frac{1.265(205 s+1)}{(128 s+1)^{2}(11.7 s+1)}
\end{array}\right]\left[\begin{array}{c}
\mu_{T} \\
\mu_{B}
\end{array}\right]} \\
& {\left[\begin{array}{l}
N_{E} \\
P_{T}
\end{array}\right]=\left[\begin{array}{cc}
\frac{1.483 s(150 s+1)}{\left(632 s^{2}+40 s+1\right)(2.7 s+1)} & \frac{2.116(457 s+1)}{(221 s+1)^{2}(21.8 s+1)} \\
-0.828\left(0.01+\frac{0.99}{97 s+1}\right) & \frac{1.649(275 s+1)}{(168 s+1)^{2}(11.5 s+1)}
\end{array}\right]\left[\begin{array}{c}
\mu_{T} \\
\mu_{B}
\end{array}\right]}
\end{aligned}
$$

First,it trains with PSO-RBF algorithm, and compares with traditional RBF algorithm.error curves are showed as figure 2.

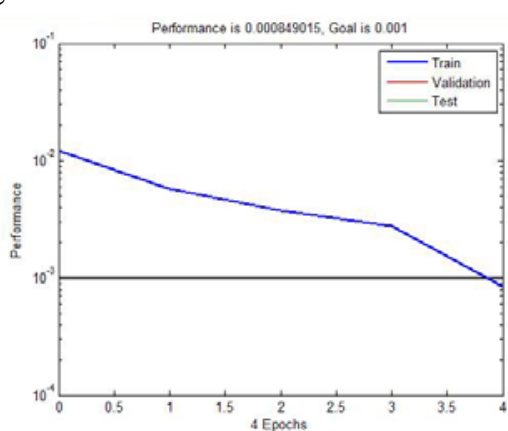

(a)

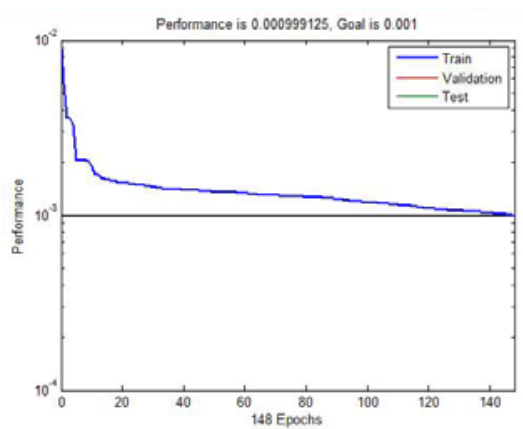

(b)

Fig. 2 network model curve of the training error

Figure 2.(a)shows PSO-RBF algorithm,it achieves the goal 0.001 by training 4 steps;figure 2.(b)Shows traditional RBF algorithm, it achieves the goal 0.001 by training 148 steps.

Then,test the generalization ability of the network.generalization curve are showed as figure 3 . 


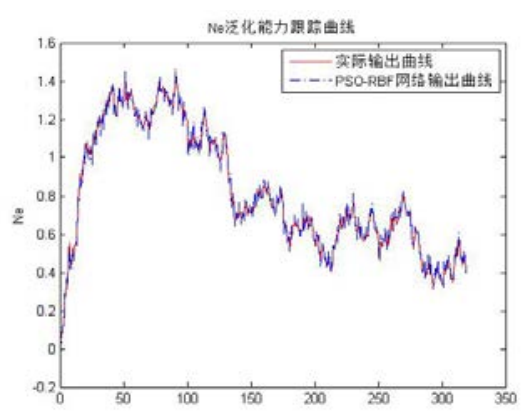

(a)

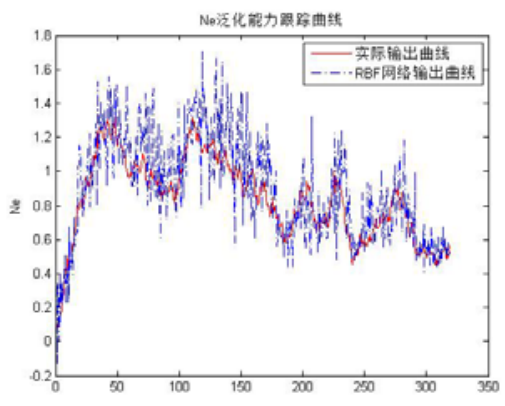

(b)

Fig. 3 generation ability curve of network

Figure 3.(a) shows optimized generalization curve by PSO.figure 3.(b) shows generalization curve which is not optimized by PSO.

On the basis of the above results,PSO-RBF algorithm is superior to traditional RBF in the matter of speed of convergence and model recognization ability.

\section{PSO-RBF GPC response simulation}

In order to verify the robustness of PSO-RBF GPC to unit variable load control,in this paper,it makes computer simulation experiments for control object at charged load with PSO-RBF neural network generalized predictive control.in this paper,elect control parameter sampling period of 10s, forecast the time domain 10 , control time domain take 3 , diffusion coefficient take 0.7 , model error correction parameter is 0.5 .pressure setting: $P_{\mathrm{t}}=18 \mathrm{MW}$,power setting: $N_{\mathrm{e}}=300 \mathrm{MW}$.at $100 \%$ load when the unit achieved stable control, keep predition model and the control parameters unchanged,then on the time 250s the condition switch to $70 \%$ load, observe its control performance $^{[6]}$.

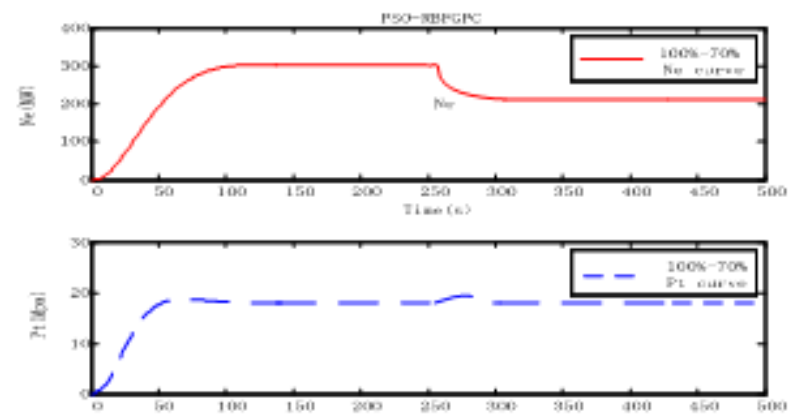

Fig. 4 output curve of Ne and Pt with PSO-RBFGPC variable load control

Figure 4 shows the output curve of $N_{\mathrm{e}}$ and $P_{\mathrm{t}}$ with PSO-RBF generalized predictive control strategy.the simulation result shows this controled method can make the system outputs track settings rapidly and smoothly,and has a good robustness and adaptability for model indeterminacy.

For comparison the effect of PSO-RBFGPC, it makes the control method compare with conventional PID control strategy. At $100 \%$ load,PID controller parameters: $\mathrm{K}_{\mathrm{bp}}=100, \mathrm{~K}_{\mathrm{bi}}=0.02$, $\mathrm{K}_{\mathrm{bd}}=10000 ; \mathrm{K}_{\mathrm{vp}}=-12, \mathrm{~K}_{\mathrm{vi}}=-0.006, \mathrm{~K}_{\mathrm{vd}}=0$.keep the control parameters unchanged and switch the condition to $70 \%$ when $\mathrm{t}$ is 250 s and observe the simulation curve.

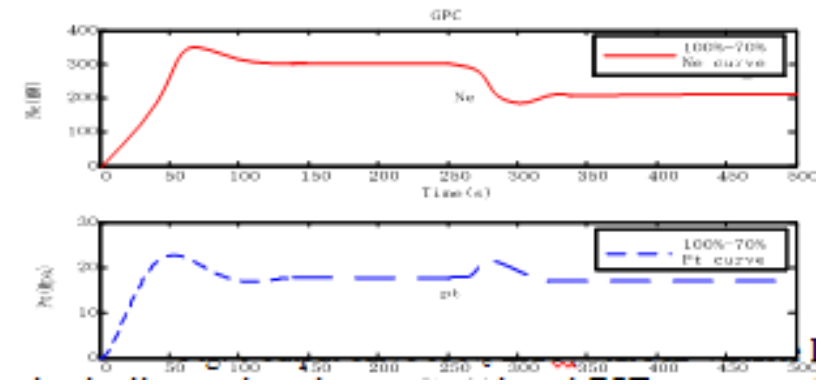

Fig. 5 output curve of $\mathrm{N}_{\mathrm{e}}$ and $\mathrm{P}_{\mathrm{t}}$ with PID variable load control

Simulation results indicate that the conventional PID strategy has good dynamic performance and 
stable performance at $100 \%$ load.however,when $\mathrm{t}$ is $250 \mathrm{~s}$, the condition switching to $70 \%, N_{\mathrm{e}}$ has larger overshoot,steam pressure changes a lot.this is not in admission.so comparing to the PSO-RBF GPC, PID strategy has less robustness and less anti-interference performance.

\section{Conclusion}

Based on the characteristics of the large-scale generating unit, the article adopts PSO-RBF algorithm to train neural network. training speed and accuracy are improved,and it can be faster to complete identification for this multi-variable nonlinear system.it works out generalized predictive control rata with the parameters of trained PSO-RBF network.compared with Diophantine equations,this method reduces computation burden and speeds up the calculation processes.the simulation results show that the algorithm of PSO-RBF neural network generalized predictive control can make the system outputs track settings rapidly and smoothly, and it has a good robustness.in general,comparing with conventional control strategy,it is more effective.

\section{References}

[1] Yu Hongjie. The coordinated control system for fossil-fired boiler-turbine unit. Baoding: North China Electric Power University, 2010

[2] Liu Yongke, Zhao Wenzhong, He Tongdi. RBF netual network with parameters optimized by particle swarm optimization algorithm of harmonic detection. Automation \& Instrumentation, 2011; 6: 133-136

[3] Li Lin , Li Jianbing, Niu Pengchao. Optimization approach based on particle swarm for RBF neural network. Journal of Shandong Electric Power College. 2010, 13: 51-53

[4] Li Hui, Cai Min, Tan Liang. Algorithm research of RBF neural network based on improved PSO. Fire Control \& Command Control, 2012; 37(2): 144-150

[5] Ling Hujun, Li Ning. Generalized predictive control srategy of boiler-turbine coordination in thermal generating unit. Control and Decision, 2013; 18: 31-36

[6] Ling Hujun, Li Ning. Study of GA-RBF neural network generalized predictive control strategy for robust control of complex system. Journal of inner mongolia university of technology, 2011; 30(3): 364-370 